the diagnostic criteria for OCD. Both illnesses appear to have neurobiological similarities but a differing course and clinical response to pharmacological treatments.

Despite this, research into optimal management of adults with co-occurring TS or other tic disorders and OCD remains sparse. Comorbidities, are known to be poor predictor of response to selective serotonin reuptake inhibitors (SSRI) monotherapy in OCD and are often associated with treatment-refractory OCD. Similarly SSRI monotherapy in patients with OCD and comorbid TS can sometimes worsen motor tics (1 in 2000) and fail to improve OCD symptoms. In this review, we aim to evaluate evidence on the management of patients with co-occurring TS and OCD and address an important knowledge gap in clinical practice.

Method. This review was conducted in accordance with PRISMA Guidelines. We performed a search using PubMed, Cochrane Library and PsychINFO using the following Boolean Input "Tourettic-OCD" OR "tic-related OCD" OR ((OCD OR "obsessive-compulsive" OR "obsessive compulsive") AND (Tourette OR “Tourette's" OR Tourettes OR tic))". The search was conducted until January 2020. We then screened the articles of systematic reviews to extract additional studies from their reference lists.

Result. 1888 studies were identified, of which 15 clinical trials were included in our systematic review. The presence of tics in patients with OCD are a major predictor for treatment-refractory OCD and a lack of improvement following monotherapy with SSRIs. Dual therapy with an SSRI and antipsychotics (particularly risperidone) are associated with improved outcomes in OCD patients with tics and TS patients with obsessive-compulsive symptoms. However, conjoint therapy with neuroleptics and SSRIs was only investigated when OCD burden was unsatisfactory following SSRI monotherapy.

Conclusion. There are clinical implications when a patient with OCD also has a chronic tic disorder. The findings indicate the need for further research, particularly in the form of a larger cohort in randomised controlled trials, to determine when it is best to initiate patients with OCD and comorbid tic disorders on a dual antipsychotic-SSRI management strategy. Further evidence should also be done to determine other characteristics that predict an improvement to conjoint SSRI/neuroleptic therapy for effective symptom reduction.

\section{Obsessive compulsive disorder in coroners' reports}

Himanshu Tyagi ${ }^{1 \star}$ and Lisa Quigley ${ }^{2}$

${ }^{1}$ UCL Division of Psychiatry, UCL Division of Neurology, UCLH and

${ }^{2}$ MSc Student, UCL Division of Psychiatry

${ }^{*}$ Corresponding author.

doi: 10.1192/bjo.2021.789

Aims. The frequency and burden of suicidality in obsessivecompulsive and related disorders have historically been underreported, despite research pointing to a significant association between OCD and suicidality. Likewise, OCD is frequently undiagnosed or misdiagnosed. This study looks at coroners' reports relating to suicides in UK, Australia and Canada in order to:

Explore characteristics of suspected or confirmed cases of OCD in coroners' reports OCD

Identify recurring themes in the reports

Method. 1869 publicly available coroners' reports were accessed from England $(n=200)$, Scotland $(n=128)$, Canada $(n=680)$ and Australia $(\mathrm{n}=861)$. Reports were screened in order to identify individuals who had either a diagnosis of OCD $(n=16)$, a diagnosis of a related condition $(\mathrm{n}=4)$, or indications of possible undiagnosed OCD $(n=12)$. Wherever possible, demographic and psychiatric characteristics were extracted for statistical analysis. Qualitative thematic analysis was carried out on selected reports.

Result. 32 cases of interest were identified from analysis of coroners' reports of suicides that took place between the years of 2000 and 2020. Breakdown by country was as follows:

United Kingdom: $\mathrm{n}=6(1.8 \%$ of total reports analysed from United Kingdom)

Canada $\mathrm{n}=3(0.4 \%$ of total reports analysed from Canada)

Australia $\mathrm{n}=23 \quad(2.7 \%$ of total reports analysed from Australia)

Among those with possible undiagnosed OCD, common experiences were fear of causing harm, intrusive thoughts of guilt and shame, and compulsive checking and/or reassurance seeking. Further themes included: misdiagnosis, failings in mental health care, stigma and discrimination.

Conclusion. Individuals with OCD are thought to be up to ten times more likely to die by suicide, with this risk increasing in the presence of psychiatric comorbidities. However, OCD remains underdiagnosed, and this may be reflected in the relatively low number of suicides identified for this study where OCD was diagnosed before death. The low numbers may also point to a tendency among both coroners and healthcare professionals to underestimate the association between OCD and suicidality.

Qualitative analysis of the coroners' reports identified a theme of intolerable distress. This distress was documented most extensively in reports where OCD was strongly indicated but never diagnosed, highlighting the impact of missed, late or incorrect diagnosis.

Notably, nearly all of the reports reveal repeated attempts by the individual to seek help. Despite this, many experienced stigma, mental health service failings and missed opportunities for help in the months preceding their deaths.

\section{Disability and functional outcomes following STN, $\mathrm{VC} / \mathrm{VS}$, and combined deep brain stimulation in obsessive compulsive disorder}

Himanshu Tyagi

UCL Institute of Neurology, Queen Square, The National Hospital for Neurology and Neurosurgery

doi: 10.1192/bjo.2021.790

Background. OCD severity scores mostly convey information within the domain of clinical conceptualisations. To capture the full impact of any new intervention it is crucial to measure its impact on disability. For this purpose we captured prospective data on changes in disability, function and impairments with multiple tools throughout the UCL-MRC trial of Deep brain stimulation (DBS) for Obsessive Compulsive Disorder (OCD) between 2013-2017. The clinical and cognitive outcomes from the trial have already been reported in 2019 . We hypothesized a concomitant improvement in perceived and observed indicators of disability with clinical improvement in OCD symptoms. This is a preliminary report of the disability outcome data from the trial.

Method. Six patients with severe treatment resistant OCD were recruited for this study from the NHS England OCD Specialist Service. Eligible participants were offered lesion surgery 